\title{
Counter-Matching: A Stratified Nested Case-Control Sampling Method
}

\author{
Bryan Langholz \\ Department of Preventive Medicine, \\ University of Southern California, School of Medicine, \\ 2025 Zonal Ave, Los Angeles, California 90033-9987, U.S.A. \\ Ørnulf Borgan \\ Institute of Mathematics, P.O. Box 1053 Blindern, \\ University of Oslo, N-0316 Oslo 3, Norway
}

April 1993

\begin{abstract}
A new type of nested case-control sampling is presented in which the sampled risk sets include the failure and random samples from "sampling strata" defined by covariate information available for all cohort subjects. This sampling may be non-representative in that the proportion sampled from each stratum need not be representative of the entire risk set. Asymptotic relative efficiency comparisons indicate that this type of sampling has superior efficiency to simple nested case-control sampling in situations of practical interest. A simple extension of the method is given which allows for non-representative sampling of failures. Analysis of stratified sampled data may be performed using standard conditional logistic likelihood software which allows for an "offset" in the model.
\end{abstract}

\section{Introduction}

Epidemiologic cohort studies of a rare disease require many subjects and/or long follow up periods in order to accumulate enough diseased subjects to have sufficient power to explore the variation in rates across various factors of interest. But, because they are large studies, collecting high quality covariate information on each subject is an expensive task. Thus, often only enough information is collected on the full cohort to allow one to draw a simple nested case-control sample (Thomas, 1977; Oakes, 1981) in which each diseased "case" is matched to a random sample of "controls" from those at risk at the case failure time. Additional detailed covariate information is then gathered for subjects in this sample to perform a proper analysis of the data. But, suppose there is additional information known for a significant portion of the cohort. For example, suppose exposure information has been gathered for all members of the cohort and it is desired to collect additional information on a sample of the cohort in order to assess the role of potential confounders or to study interactions of the exposure with other risk factors. Another possibility is that a crude measure of exposure has been gathered

\footnotetext{
${ }^{0}$ Key words and phrases. Asymptotic efficiency; Cohort study; Case-control study; Design of medical study; Epidemiology; Martingale; Survival analysis
} 
for most of the cohort members and researchers wish to collect more precise exposure data on a much smaller sample of the cohort.

In drawing a nested case-control sample only the at-risk status of the cohort members is used. It is easy to imagine that incorporating other covariate information into the sampling process might lead to a more informative sample. This concept has been discussed in the context of grouped data situations and logistic regression in White (1982), Breslow and Cain (1988), Cain and Breslow (1988), Weinberg and Wacholder (1990) and Weinberg and Sandler (1991). Here, we develop the theory and present large sample efficiency results for stratified sampling of controls in a modification of simple nested case-control sampling from a cohort using which we call "counter-matching." As it is often the situation that there are a relatively small number of cases, we will assume for the time being, that all cases are to be used and that sampling is of the risk sets at the failure times in the cohort. We discuss non-representive sampling of cases in Section 4.

We assume the Cox proportional hazards model (Cox, 1972) where the conditional hazard for a subject with vector of covariates $Z(t)$ at time $t$ may be given as

$$
\lambda_{0}(t) \exp \left\{\beta_{0}^{\prime} Z(t)\right\} \text {. }
$$

Let $t_{j}$ be the $j$ th ordered failure time and $i_{j}$ be the index of the failure at time $t_{j}$. At $t_{j}$ a counter-matched sampled risk set $\widetilde{\mathcal{R}}\left(t_{j}\right)$ of size $m$, with $m-1$ controls and 1 case, is drawn as follows: Each person in the risk set, including the case, is classified into one of, say, $L$ sampling strata. This classification cannot be based on case-control status. More precisely, if someone other than the actual case had been the case, it would not have resulted in a change in the way classification is done. Then $\widetilde{\mathcal{R}}\left(t_{j}\right)$ is to consist of $m_{l}>0$ subjects from the $n_{l}\left(t_{j}\right)$ at risk individuals in stratum $l$ where the $m_{l}$ are chosen in advance and do not need to reflect the representation of stratum $l$ in the full risk set. When actually performing the sampling, one randomly samples, without replacement, $m_{l}$ controls from stratum $l$ except for the case's stratum from which one samples only $m_{l}-1$ controls. The case is always included in the sample so that there are a total of $m_{l}$ from stratum $l$. Let $A_{k}\left(t_{j}\right)$ be the sampling stratum for subject $k$ at time $t_{j}$ and $w_{k}\left(t_{j}\right)=n_{A_{k}\left(t_{j}\right)}\left(t_{j}\right) / m_{A_{k}\left(t_{j}\right)}$. Then, as will be shown in Section 2 , the partial likelihood for the sampled data set is given by

$$
\mathcal{L}(\beta)=\prod_{t_{j}}\left[\frac{\exp \left\{\beta^{\prime} Z_{i_{j}}\left(t_{j}\right)\right\} w_{i_{j}}\left(t_{j}\right)}{\sum_{k \in \widetilde{\mathcal{R}}\left(t_{j}\right)} \exp \left\{\beta^{\prime} Z_{k}\left(t_{j}\right)\right\} w_{k}\left(t_{j}\right)}\right] .
$$

This has the form of the usual "conditional logistic" likelihood where the contribution of a subject from stratum $l$ is weighted by the inverse of the proportion in the sampled risk set from stratum $l$. The partial likelihood (2) has "basic likelihood properties" by which we mean that expectation of the score evaluated at $\beta_{0}$ equals zero, and the expected information matrix at $\beta_{0}$ equals the covariance matrix of the the score. Standard conditional logistic regression fitting algorithms may be used simply by including a subject's $\log$ weight, $\log w_{i}\left(t_{j}\right)$, as an "offset" in the model. This feature is currently available in many software packages.

As an example, consider a cohort in which a dichotomous exposure is known for all at risk subjects at each failure time. Additional information is to be collected on a sample and might include precise exposure measurements, confounder data, and other known or potential exposure information. In 1:1 counter-matched sampling, each exposed case is matched to a control randomly selected from those subjects who were at risk and unexposed at the case's 
failure time. Similarly, each unexposed case is matched to an exposed control. Thus, the term counter-matching is descriptive of this procedure as it is essentially opposite of "matching" on exposure. Analysis of the sampled data is performed using (2) with $w_{i}\left(t_{j}\right)$ the number of exposed at risk at the failure time if subject $i$ is exposed at the failure time or the number of unexposed if $i$ is unexposed.

An appealing feature of this method is that the full cohort information about the sampling stratification variable is summarized into the sample in the following sense. If the only covariates in the model are functions of the $A_{j}(t)$, it may be easily shown that (2) is the full cohort partial likelihood. Also, we note that there is no requirement that the sampling strata be included in the model as covariates for model comparison statistics, such as the likelihood ratio test between nested models, to be valid.

\section{Model and partial likelihood}

In this section we define a model for counter-matching and derive the partial likelihood (2) based on this model.

We fix throughout a time interval $[0, \tau]$, and following the counting process formulation of the Cox model as given by Andersen and Gill (1982), we let $N_{i}, Y_{i}$, and $Z_{i}$ be the counting, censoring, and covariate processes for the $i$ th subject, $i=1, \ldots, n$. Moreover, we let $A_{i}$, which may be a function of $Z_{i}$, be one of $L$ sampling stratum indicators. While any convenient index set with $L$ elements is valid, for ease of exposition let $A_{i}(t) \in\{1, \ldots, L\}$. At time $t$ the risk set is $\mathcal{R}(t)=\left\{i: Y_{i}(t)=1\right\}$, and the number of individuals at risk is $n(t)=|\mathcal{R}(t)|=\sum Y_{i}(t)$. As is usual, we assume that there is a non-decreasing family of $\sigma$-algebras $\left(\mathcal{H}_{t}\right)_{t \in[0, \tau]}$ such that the $N_{i}$ are adapted to $\left(\mathcal{H}_{t}\right)$ and the $Y_{i}, Z_{i}$, and $A_{i}$ are predictable with respect to $\left(\mathcal{H}_{t}\right)$. $\mathcal{H}_{t}$ is the "cohort history" including failure time, censoring, and covariate information up to time $t$. The $\left(\mathcal{H}_{t}\right)$-intensity process $\lambda_{i}$ of $N_{i}$ is given heuristically by

$$
\lambda_{i}(t) \mathrm{d} t=\operatorname{pr}\left\{\mathrm{d} N_{i}(t)=1 \mid \mathcal{H}_{t-}\right\},
$$

where $\mathrm{d} N_{i}(t)=N_{i}\{(t+\mathrm{d} t)-\}-N_{i}(t-)$ is the increment of $N_{i}$ over the small time interval $[t, t+\mathrm{d} t)$. Assuming censoring to be independent (Andersen et al., 1993, Section III.2.2), (1) yields $\lambda_{i}$ given by

$$
\lambda_{i}(t)=Y_{i}(t) \lambda_{0}(t) \exp \left\{\beta_{0}^{\prime} Z_{i}(t)\right\} .
$$

We write $\mathcal{R}_{l}(t)=\left\{i: Y_{i}(t)=1, A_{i}(t)=l\right\}$ for the subset of $\mathcal{R}(t)$ which belongs to stratum $l$, and let $n_{l}(t)=\left|\mathcal{R}_{l}(t)\right|$ be the number of individuals in this stratum at time $t$. Then if a subject, say $i$, fails at time $t, m_{l}$ controls are randomly sampled without replacement from $\mathcal{R}_{l}(t)$ except for the failure's stratum $\mathcal{R}_{A_{i}(t)}(t)$ from which $m_{A_{i}(t)}-1$ are sampled from the

$n_{A_{i}(t)}(t)-1$ non-failures. We let $\widetilde{\mathcal{R}}(t)$ denote the sampled risk set at $t$ were a failure to occur at that time. This will consist of the failing individual together with a sampled set of controls. As a technical point, the number of controls could also depend on time. Specifically, if $n_{l}(t)<m_{l}$ for some $l$ we would sample all individuals in this stratum but, for simplicity of exposition, we will assume below that the numbers of subjects sampled from each stratum do not depend on $t$. We introduce

$$
\mathcal{P}(t)=\left\{\mathbf{r} \subset \mathcal{R}(t):\left|\mathbf{r} \cap \mathcal{R}_{l}(t)\right|=m_{l}, l=1, \ldots, L\right\},
$$


and $\mathcal{P}_{i}(t)=\{\mathbf{r} \in \mathcal{P}(t): i \in \mathbf{r}\}$. Then $\mathcal{P}(t)$ is the collection of all possible sets that may actually arise as sampled risk sets were a failure to occur at time $t$, while $\mathcal{P}_{i}(t)$ is the collection of all possible sets if subject $i$ is the failure. Note that there are $C(t) m_{A_{i}(t)} / n_{A_{i}(t)}(t)$ sets in $\mathcal{P}_{i}(t)$ where

$$
C(t)=\prod_{l=1}^{L}\left(\begin{array}{c}
n_{l}(t) \\
m_{l}
\end{array}\right) .
$$

We let $\mathcal{F}_{t-}$ contain information about all observed events in the cohort as well as about the sampling of controls in $[0, t)$. Thus $\mathcal{F}_{t-}$ is $\mathcal{H}_{t-}$ augmented with the sampling information. Then we have

$$
\operatorname{pr}\left\{\tilde{\mathcal{R}}(t)=\mathbf{r} \mid \Delta N_{i}(t)=1, \mathcal{F}_{t-}\right\}=C(t)^{-1} w_{i}(t) I\left\{\mathbf{r} \in \mathcal{P}_{i}(t)\right\},
$$

where $\Delta N_{i}(t)=N_{i}(t)-N_{i}(t-)$ is the increment of $N_{i}$ at $t$, and $w_{i}(t)=n_{A_{i}(t)}(t) / m_{A_{i}(t)}$.

For each set $\mathbf{r} \in \mathcal{P}^{(m)}$, where $\mathcal{P}^{(m)}$ is the set of all subsets of $\{1,2, \ldots, n\}$ of size $m=\sum m_{l}$, we define $N_{(i, \mathbf{r})}(t)$ as the number of times in $[0, t]$ the $i$ th individual fails and the sampled risk set is chosen to be $\mathbf{r}$. Moreover, we assume that the sampling is independent in the sense that the additional knowledge of which individuals have been sampled as controls before any time $t$ do not alter the intensities of failures at $t$. Thus $\operatorname{pr}\left\{\mathrm{d} N_{i}(t)=1 \mid \mathcal{F}_{t-}\right\}=\operatorname{pr}\left\{\mathrm{d} N_{i}(t)=1 \mid \mathcal{H}_{t-}\right\}$. Informally therefore, by (3) and (5), the intensity process $\lambda_{(i, \mathbf{r})}$ of the counting process $N_{(i, \mathbf{r})}$ is given by

$$
\begin{aligned}
\lambda_{(i, \mathbf{r})}(t) \mathrm{d} t & =\operatorname{pr}\left\{\mathrm{d} N_{(i, \mathbf{r})}(t)=1 \mid \mathcal{F}_{t-}\right\}=\operatorname{pr}\left\{\mathrm{d} N_{i}(t)=1, \widetilde{\mathcal{R}}(t)=\mathbf{r} \mid \mathcal{F}_{t-}\right\} \\
& =\operatorname{pr}\left\{\mathrm{d} N_{i}(t)=1 \mid \mathcal{F}_{t-}\right\} \times \operatorname{pr}\left\{\widetilde{\mathcal{R}}(t)=\mathbf{r} \mid \Delta N_{i}(t)=1, \mathcal{F}_{t-}\right\} \\
& =\lambda_{i}(t) \mathrm{d} t C(t)^{-1} w_{i}(t) I\left\{\mathbf{r} \in \mathcal{P}_{i}(t)\right\}
\end{aligned}
$$

These heuristics, combined with (4), imply that the counting processes $N_{(i, \mathbf{r})}$, for $\mathbf{r} \in \mathcal{P}^{(m)}$ and $i \in \mathbf{r}$, have intensity processes

$$
\lambda_{(i, \mathbf{r})}(t)=Y_{i}(t) \lambda_{0}(t) \exp \left\{\beta_{0}^{\prime} Z_{i}(t)\right\} C(t)^{-1} w_{i}(t) I\left\{\mathbf{r} \in \mathcal{P}_{i}(t)\right\} .
$$

This gives a specification of the model for the sampling of controls as well as for the occurence of failures.

To derive the partial likelihood (2), we first introduce

$$
N_{\mathbf{r}}(t)=\sum_{i \in \mathbf{r}} N_{(i, \mathbf{r})}(t)
$$

for the process counting the number of times the sampled risk set equals $\mathbf{r}$ in $[0, t]$, and note that its intensity process, by (6), is

$$
\lambda_{\mathbf{r}}(t)=\sum_{j \in \mathbf{r}} \lambda_{0}(t) \exp \left\{\beta_{0}^{\prime} Z_{j}(t)\right\} C(t)^{-1} w_{j}(t) I\{\mathbf{r} \in \mathcal{P}(t)\}
$$

Then we factorise the intensity processes $\lambda_{(i, \mathbf{r})}$, not as just above (6), but as

$$
\lambda_{(i, \mathbf{r})}(t)=\lambda_{\mathbf{r}}(t) \pi_{t}(i \mid \mathbf{r}),
$$


where, by (6) and (8),

$$
\pi_{t}(i \mid \mathbf{r})=\frac{\exp \left\{\beta_{0}^{\prime} Z_{i}(t)\right\} w_{i}(t)}{\sum_{j \in \mathbf{r}} \exp \left\{\beta_{0}^{\prime} Z_{j}(t)\right\} w_{j}(t)}
$$

for $i \in \mathbf{r}$ and $\mathbf{r} \in \mathcal{P}(t)$. Note that (9) has the interpretation of the conditional probability of the $i$ th individual failing at $t$, given $\mathcal{F}_{t-}$ and that there is a failure among individuals in the set $\mathbf{r}$ at $t$. The partial likelihood (2) is then obtained by multiplying together conditional probabilities of the form (9) for each failure time and sampled risk set.

In Appendix 1 we show that (2) has basic likelihood properties, i.e. that the score vector has expectation zero, and that its covariance matrix equals the expected information matrix. From these properties, the usual Taylor series argument may be used to show that the maximum partial likelihood estimator $\hat{\beta}$, obtained by maximizing (2), asymptotically has a multivariate normal distribution with mean $\beta_{0}$ and covariance matrix $n^{-1} \Sigma^{-1}$. This covariance matrix may be estimated in the usual way by the inverse of the observed information matrix. An expression for the asymptotic information matrix $\Sigma$ is derived in Appendix 2.

\section{Asymptotic relative efficiencies}

This section presents a large sample comparison of simple nested case- control sampling to the stratified version using counter-matching in two situations where the new design shows promise: when sampling strata are based (1) on a surrogate measure of exposure and true exposure measurements are collected for the sample, and (2) on exposure and confounder information is collected for the sample. We first give a generic model which encompasses the special cases we will use in our large sample comparisons for both these situations. Let $Z_{1}$ and $Z_{2}$ be dichotomous with values zero and one. It is assumed that $Z_{1}$ is known for the entire cohort and $Z_{2}$ is collected only for the sample. For the simple nested case-control design, sampled risk sets of size $m$ consist of the failure and $m-1$ controls randomly sampled without regard to " $Z_{1}$ " status. For the counter-matched sample, $m_{j}$ subjects are sampled, with the failure sampled with probability one, from those with $Z_{1}=j$ with $m_{0}+m_{1}=m$. Let the intensity process for an subject with covariates $Z_{1}$ and $Z_{2}$ be specified as

$$
\lambda(t)=Y(t) \lambda_{0}(t) \exp \left(Z_{1} \beta_{1}+Z_{2} \beta_{2}\right),
$$

c.f. (4).

We assume that the joint distribution of $Z_{1}$ and $Z_{2}$ for those at risk remain constant over time with $\pi_{i j}=\operatorname{pr}\left(Z_{1}=i, Z_{2}=j \mid Y(t)=1\right), \pi_{i}=\operatorname{pr}\left(Z_{1}=i \mid Y(t)=1\right)$, and $\pi_{\cdot i}=\operatorname{pr}\left(Z_{2}=i \mid Y(t)=1\right)$. Subjects in the cohort are assumed to arise as independent and identically distributed realizations from the covariate and censoring distributions with failure time distributions determined by the associated hazard functions. In Appendix 3 we derive, for both sampling designs, explicit expressions for a quantity proportional to the asymptotic information matrix under these assumptions. As in Goldstein and Langholz (1992, Example $6.3 \mathrm{~b})$, it turns out that the asymptotic relative efficiencies do not depend on the failure rate.

\subsection{Counter-matching on a surrogate measure of exposure}

When the exposure of interest is expensive to collect for all cohort members but there is a surrogate measure that may be cheaply obtained, counter-matching on the surrogate measure 
Table 1: Asymptotic relative efficiencies of counter-matched versus simple nested case-control sampling when stratification is based on a surrogate measure of exposure with specificity $1-\beta$ and sensitivity $1-\alpha^{a}$.

\begin{tabular}{|c|c|c|c|c|c|c|c|c|}
\hline \multicolumn{9}{|c|}{ (a) $\pi_{\cdot 1}=0.05$} \\
\hline & \multicolumn{4}{|c|}{$\begin{array}{c}1: 1 \text { matching }(m=2) \\
1-\beta\end{array}$} & \multicolumn{4}{|c|}{$\begin{array}{c}1: 3 \text { matching }(m=4) \\
1-\beta\end{array}$} \\
\hline $1-\alpha$ & 0.5 & 0.7 & 0.9 & 1.0 & 0.5 & 0.7 & 0.9 & 1.0 \\
\hline 0.40 & 1.00 & 0.96 & 1.06 & 1.18 & 1.00 & 0.97 & 0.98 & 0.99 \\
\hline 0.50 & 1.00 & 1.07 & 1.29 & 1.46 & 1.00 & 1.02 & 1.06 & 1.10 \\
\hline 0.70 & 1.00 & 1.27 & 1.72 & 2.03 & 1.00 & 1.10 & 1.23 & 1.31 \\
\hline 0.80 & 0.99 & 1.38 & 1.94 & 2.31 & 1.00 & 1.14 & 1.31 & 1.41 \\
\hline 0.90 & 0.99 & 1.47 & 2.15 & 2.58 & 1.00 & 1.18 & 1.39 & 1.51 \\
\hline 0.95 & 0.99 & 1.52 & 2.25 & 2.72 & 1.00 & 1.20 & 1.43 & 1.56 \\
\hline 1.00 & 0.98 & 1.57 & 2.36 & 2.86 & 1.00 & 1.21 & 1.47 & 1.61 \\
\hline \multicolumn{9}{|c|}{ (b) $\pi_{\cdot 1}=0.5$} \\
\hline & \multirow{2}{*}{\multicolumn{4}{|c|}{$\begin{array}{c}1: 1 \text { matching }(m=2) \\
1-\beta\end{array}$}} & \multirow{2}{*}{\multicolumn{4}{|c|}{$\begin{array}{c}1: 3 \text { matching }(m=4) \\
1-\beta\end{array}$}} \\
\hline & & & & & & & & \\
\hline $1-\alpha$ & 0.5 & 0.7 & 0.9 & 1.0 & 0.5 & 0.7 & 0.9 & 1.0 \\
\hline 0.40 & 0.99 & 0.95 & 0.97 & 1.00 & 1.00 & 0.98 & 0.97 & 0.97 \\
\hline 0.50 & 1.00 & 1.03 & 1.13 & 1.20 & 1.00 & 1.01 & 1.02 & 1.03 \\
\hline 0.70 & 0.98 & 1.16 & 1.41 & 1.56 & 1.00 & 1.05 & 1.12 & 1.15 \\
\hline 0.80 & 0.95 & 1.21 & 1.53 & 1.71 & 0.99 & 1.07 & 1.16 & 1.21 \\
\hline 0.90 & 0.91 & 1.25 & 1.64 & 1.86 & 0.98 & 1.09 & 1.20 & 1.26 \\
\hline 0.95 & 0.88 & 1.26 & 1.69 & 1.93 & 0.98 & 1.10 & 1.22 & 1.29 \\
\hline 1.00 & 0.86 & 1.27 & 1.74 & 2.00 & 0.97 & 1.11 & 1.24 & 1.31 \\
\hline
\end{tabular}

${ }^{a}$ For $m_{0}=m_{1}$ and $\exp \left(\beta_{2}\right)=2$.

and evaluating the true exposure for this sample is an attractive alternative to simple random sampling. In the $m=2$ case, counter-matching on a reasonably good surrogate would lead a higher proportion of exposure discordant pairs than the simple design so that one might expect better efficiency with the former design. In the context of the setting above, let $Z_{2}$ be the true exposure measurement and $Z_{1}$ be the surrogate which is assumed to be uninformative about disease rates after accounting for $Z_{2}$, i.e., $\beta_{1}=0$ in model $(10)$. We are interested in comparing the variance of $\hat{\beta}_{2}$ for the two designs.

Let $1-\alpha=\pi_{11} / \pi_{\cdot 1}$ be the sensitivity and $1-\beta=\pi_{00} / \pi_{\cdot 0}$ be the specificity of the surrogate for predicting the true exposure. Solving for the $\pi_{i j}$ in terms sensitivity, specificity, and probability of true exposure, $\pi_{\cdot 1}$, asymptotic variances were calculated using equations (25) and (26) in Appendix 3 and the asymptotic relative efficiency was computed as the ratio of the variances of $\widehat{\beta}_{2}$ for simple compared to counter-matched design. Selected values for the "balanced" counter-matched design, i.e. when $m_{0}=m_{1}$, and $\exp \left(\beta_{2}\right)=2$ are given in Table 1. Even when the surrogate is quite a poor predictor of the true exposure, substantial 
gains in efficiency are realized by counter-matching on the surrogate. As the pattern of relative efficiencies in Table 1 indicates, for sensitivities and specificities both greater than 0.5 , relative efficiencies increase with increasing sensitivity or specificity but the increase is more marked with increasing specificity than with sensitivity. In fact, this difference becomes more pronounced with increasing $\exp \left(\beta_{2}\right)$ and suggests that high specificity is more important than high sensitivity. Comparing the $\pi_{.1}=0.05$ to 0.5 portions of Table 1 , it is apparent that efficiency gains are greater for rare exposures than common ones; this might be expected given the efficiency behavior of simple nested case-control sampling (Breslow et al., 1983). We note that when $1-\alpha=1-\beta=1$, the surrogate measure predicts the true exposure exactly so that these entries in Table 1 are the relative efficiency of simple sampling to the full cohort; cf. the last paragraph in Section 1. Thus, for $\pi_{1}=0.05$ and $\exp \left(\beta_{2}\right)=2$, while a single randomly sampled control yields approximately $35 \%$ efficiency relative to the full cohort, a single counter-matched control, stratified by a surrogate with $90 \%$ specificity and $95 \%$ sensitivity, is nearly $80 \%$ efficient. In fact, to achieve the level of efficiency of such a counter-matched sample would require seven randomly sampled controls.

When $\exp \left(\beta_{2}\right)=1$ and $m=2$, the asymptotic relative efficiency is symmetric in sensitivity and specificity, independent of the probability of exposure $\pi_{.1}$, and is given by $2\{(1-\alpha)(1-$ $\beta)+\alpha \beta\}$. This may be easily derived by straightforward manipulation of expressions for the $(2,2)$ th entry in $(25)$ and $(26)$.

\subsection{Counter-matching on exposure}

In a model analogous to that considered by Breslow and Cain (1988) when evaluating their two stage sampling procedure for unconditional logistic regression, let $Z_{1}$ be an "exposure" variable, assumed to be known for the full cohort and which will serve as the sampling stratification variable, and $Z_{2}$ be a "confounder" variable to be collected for the sampled subjects. We measure the correlation between $Z_{1}$ and $Z_{2}$ by the odds ratio $\theta=\left(\pi_{11} \pi_{00}\right) /\left(\pi_{10} \pi_{01}\right)$.

We are interested in comparing the variances of $\widehat{\beta}_{1}$ after controlling for the effect of $Z_{2}$ using the two sampling designs. One might expect the counter-matched design to perform better than the simple design because the marginal information about the exposure is "carried along" into the sample. It is also of interest to compare the variances of $\widehat{\beta}_{3}$ for a model with an interaction term $Z_{1} Z_{2} \beta_{3}$ added in (10), assuming that $\beta_{3}=0$.

Solving for the $\pi_{i j}$ in terms of the odds ratio, $\theta$, and the probabilities of the exposure and confounder variables, $\pi_{1}$. and $\pi_{\cdot 1}$, asymptotic variances were calculated using equations (25) and (26) in Appendix 3. Table 2 gives asymptotic relative efficiencies of the balanced counter-matced design relative to simple nested case-control sampling. These are given for exposure probability $\pi_{1}=0.05$ and probability of being positive for the confounder $\pi_{\cdot 1}=0.3$ and various values of $m, \exp \left(\beta_{1}\right), \exp \left(\beta_{2}\right)$ and $\theta$. In every case, counter-matching results in substantial gains in efficiency for estimating $\beta_{1}$ after controlling for $Z_{2}$, and for the estimation of $\beta_{3}$. For $m=2$, there are large losses of efficiency for estimating $\beta_{2}$ after controlling for $Z_{1}$ but, since $Z_{2}$ is a confounder in the model, a precise estimate of its effect is not important. We also computed the asymptotic relative efficiencies of the balanced design compared to the stratified sample with optimal $m_{0}$ and $m_{1}$ for $m=4$ or 8 . For all combinations of parameters used in Table 2, the there was very little difference between the balanced and optimal designs.

We also considered the situation where the observed relative risk for exposure is explained by the confounder. In this situation, $\beta_{1}=0$ and $\beta_{2}$ was chosen to yield a a marginal relative risk for exposure of $\exp \left(\beta_{1}^{*}\right)=1.5$ or 2 when full cohort data is used to estimate $\beta_{1}$ without 
Table 2: Asymptotic relative efficiencies of stratified versus simple nested case-control sampling when the exposure relative risk $\exp \left(\beta_{1}\right)$ is 2 or $4^{a}$.

\begin{tabular}{|c|c|c|c|c|c|c|c|}
\hline \multicolumn{8}{|c|}{ (a) $\exp \left(\beta_{1}\right)=2$} \\
\hline & & \multicolumn{3}{|c|}{$1: 1$ matching $(m=2)$} & \multicolumn{3}{|c|}{$1: 3$ matching $(m=4)$} \\
\hline$e^{\beta_{2}}$ & $\theta$ & $\widehat{\beta}_{1}$ & $\widehat{\beta}_{2}$ & $\widehat{\beta}_{3}$ & $\widehat{\beta}_{1}$ & $\widehat{\beta}_{2}$ & $\hat{\beta}_{3}$ \\
\hline 0.2 & 0.2 & 2.65 & 0.45 & 1.50 & 1.73 & 0.83 & 1.16 \\
\hline 0.2 & 0.5 & 2.75 & 0.45 & 1.42 & 1.72 & 0.84 & 1.16 \\
\hline 0.2 & 1.0 & 2.83 & 0.45 & 1.32 & 1.71 & 0.85 & 1.17 \\
\hline 0.2 & 2.0 & 2.86 & 0.45 & 1.21 & 1.68 & 0.86 & 1.18 \\
\hline 0.2 & 5.0 & 2.63 & 0.43 & 1.11 & 1.59 & 0.87 & 1.23 \\
\hline 1.0 & 0.2 & 2.46 & 0.25 & 2.00 & 1.58 & 0.75 & 1.51 \\
\hline 1.0 & 0.5 & 2.75 & 0.30 & 1.64 & 1.60 & 0.77 & 1.47 \\
\hline 1.0 & 1.0 & 2.86 & 0.35 & 1.42 & 1.61 & 0.79 & 1.43 \\
\hline 1.0 & 2.0 & 2.73 & 0.38 & 1.31 & 1.60 & 0.80 & 1.41 \\
\hline 1.0 & 5.0 & 2.23 & 0.37 & 1.35 & 1.54 & 0.80 & 1.42 \\
\hline 5.0 & 0.2 & 2.56 & 0.26 & 2.19 & 1.55 & 0.67 & 1.84 \\
\hline 5.0 & 0.5 & 2.85 & 0.35 & 1.75 & 1.71 & 0.71 & 1.60 \\
\hline 5.0 & 1.0 & 2.80 & 0.42 & 1.62 & 1.80 & 0.73 & 1.46 \\
\hline 5.0 & 2.0 & 2.58 & 0.46 & 1.62 & 1.82 & 0.74 & 1.37 \\
\hline 5.0 & 5.0 & 2.24 & 0.49 & 1.74 & 1.78 & 0.74 & 1.32 \\
\hline \multicolumn{8}{|c|}{ (b) $\exp \left(\beta_{1}\right)=4$} \\
\hline & & \multicolumn{3}{|c|}{$1: 1$ matching $(m=2)$} & \multicolumn{3}{|c|}{$1: 3$ matching $(m=4)$} \\
\hline$e^{\beta_{2}}$ & $\theta$ & $\widehat{\beta}_{1}$ & $\widehat{\beta}_{2}$ & $\widehat{\beta}_{3}$ & $\widehat{\beta}_{1}$ & $\widehat{\beta}_{2}$ & $\widehat{\beta}_{3}$ \\
\hline 0.2 & 0.2 & 4.06 & 0.70 & 1.90 & 2.23 & 0.90 & 1.30 \\
\hline 0.2 & 0.5 & 4.15 & 0.71 & 1.81 & 2.23 & 0.92 & 1.29 \\
\hline 0.2 & 1.0 & 4.20 & 0.71 & 1.71 & 2.21 & 0.94 & 1.29 \\
\hline 0.2 & 2.0 & 4.13 & 0.72 & 1.60 & 2.16 & 0.96 & 1.30 \\
\hline 0.2 & 5.0 & 3.63 & 0.70 & 1.52 & 2.02 & 0.99 & 1.37 \\
\hline 1.0 & 0.2 & 3.75 & 0.44 & 2.92 & 2.01 & 0.82 & 1.88 \\
\hline 1.0 & 0.5 & 4.19 & 0.54 & 2.40 & 2.07 & 0.87 & 1.78 \\
\hline 1.0 & 1.0 & 4.35 & 0.62 & 2.09 & 2.09 & 0.90 & 1.71 \\
\hline 1.0 & 2.0 & 4.15 & 0.67 & 1.93 & 2.06 & 0.93 & 1.66 \\
\hline 1.0 & 5.0 & 3.41 & 0.65 & 2.00 & 1.93 & 0.92 & 1.68 \\
\hline 5.0 & 0.2 & 3.46 & 0.43 & 3.27 & 1.89 & 0.77 & 2.34 \\
\hline 5.0 & 0.5 & 3.99 & 0.58 & 2.59 & 2.14 & 0.84 & 1.96 \\
\hline 5.0 & 1.0 & 4.07 & 0.69 & 2.36 & 2.26 & 0.87 & 1.75 \\
\hline 5.0 & 2.0 & 3.88 & 0.76 & 2.31 & 2.29 & 0.89 & 1.63 \\
\hline 5.0 & 5.0 & 3.47 & 0.80 & 2.40 & 2.23 & 0.89 & 1.56 \\
\hline
\end{tabular}

${ }^{a}$ For $m_{0}=m_{1}$ and $\pi_{1}=0.05$ and $\pi_{1}=0.30$.

Efficiencies for $\widehat{\beta}_{1}$ and $\widehat{\beta}_{2}$ based on fitting the model with no interaction term ' $Z_{1} Z_{2}$ '. 
Table 3: Asymptotic relative efficiencies of stratified versus simple nested case-control sampling when there is a marginal relative risk for exposure $\exp \left(\beta_{1}^{*}\right)$ of 1.5 or 2.0 but $\exp \left(\beta_{1}\right)=1$ after controlling for confounding ${ }^{a}$.

\begin{tabular}{|c|c|c|c|c|c|c|c|}
\hline \multicolumn{8}{|c|}{ (a) $\exp \left(\beta_{1}^{*}\right)=1.5$} \\
\hline \multirow[b]{2}{*}{$e^{\beta_{2}}$} & \multirow[b]{2}{*}{$\theta$} & \multicolumn{3}{|c|}{$1: 1$ matching $(m=2)$} & \multicolumn{3}{|c|}{$1: 3$ matching $(m=4)$} \\
\hline & & $\widehat{\beta}_{1}$ & $\widehat{\beta}_{2}$ & $\widehat{\beta}_{3}$ & $\widehat{\beta}_{1}$ & $\widehat{\beta}_{2}$ & $\widehat{\beta}_{3}$ \\
\hline 36.7 & 2.0 & 1.91 & 0.62 & 1.47 & 1.55 & 0.77 & 1.11 \\
\hline 3.7 & 4.0 & 1.54 & 0.25 & 1.20 & 1.45 & 0.66 & 1.20 \\
\hline 2.8 & 6.0 & 1.39 & 0.22 & 1.21 & 1.40 & 0.67 & 1.21 \\
\hline 2.5 & 8.0 & 1.28 & 0.21 & 1.24 & 1.38 & 0.67 & 1.22 \\
\hline \multirow[t]{3}{*}{2.3} & 10.0 & 1.21 & 0.20 & 1.27 & 1.36 & 0.67 & 1.22 \\
\hline & & \multicolumn{5}{|c|}{ (b) $\exp \left(\beta_{1}^{*}\right)=2$} & \\
\hline & & \multicolumn{3}{|c|}{$1: 1$ matching $(m=2)$} & \multicolumn{3}{|c|}{$1: 3$ matching $(m=4)$} \\
\hline$e^{\beta_{2}}$ & $\theta$ & $\widehat{\beta}_{1}$ & $\widehat{\beta}_{2}$ & $\widehat{\beta}_{3}$ & $\widehat{\beta}_{1}$ & $\hat{\beta}_{2}$ & $\widehat{\beta}_{3}$ \\
\hline 22.8 & 4.0 & 1.79 & 0.60 & 1.47 & 1.53 & 0.76 & 1.11 \\
\hline 8.1 & 6.0 & 1.54 & 0.35 & 1.41 & 1.51 & 0.66 & 1.15 \\
\hline 6.0 & 8.0 & 1.42 & 0.30 & 1.40 & 1.48 & 0.66 & 1.16 \\
\hline 5.1 & 10.0 & 1.35 & 0.28 & 1.41 & 1.46 & 0.65 & 1.17 \\
\hline
\end{tabular}

${ }^{a}$ See footnote to Table 1.

$Z_{2}$ in the model. The results for balanced stratified sampling are given in Table 3 . Once again stratified sampling offers considerable efficiency advantage for estimation of the exposure effect after controlling for the confounder.

These asymptotic relative efficiency calculations compare the two designs with comparable matching ratios. However, the number of distinct subjects in the sample, often the relevant determinant of the cost of the sampled study (Langholz and Thomas, 1991), is different for the two designs. If this is the case, the relative efficiencies underestimate the advantage of the stratified design since the stratified design requires a smaller proportion of the cohort than does the standard. This is because, when exposure is rare, exposed subjects have a higher chance of appearing in multiple counter-matched sampled risk sets than in the simple design. The difference in the proportion sampled increases with decreasing probability of exposure, increasing disease probability, and increasing matching ratio. Thus, if the relevant measure of sample size is the number of distinct subjects, the actual relative efficiencies may be substantially higher than suggested in the tables.

\section{Non-representative sampling of cases}

If the disease of interest is rare, as is often the situation in epidemiologic studies, all cases will be used in the sampled data set. But, occasionally, especially if there are many cases available and few of them are "exposed," some type of non-representative sampling of the cases may be desirable. This, of course, would be done before sampling controls since sampled risk sets 
are uninformative about $\beta_{0}$ if there is no case. Stratified sampling in which a fixed number of cases is picked from each sampling stratum, as we have described for the controls, cannot be accomodated by our methodology because the total number of cases in each sampling stratum for the entire study period is not in $\mathcal{F}_{t-}$ for $t<\tau$. We propose an approximation to this type of sampling in which the decision to include a case is determined by a Bernulli trial with probability depending on the sampling stratum of the case and chosen to yield the desired proportions of cases from each stratum. A stratified sample of the controls is picked as before for each included case.

A simple generalization of the hazard model (6) is required to accomodate this type of sampling. If a failure were to occur at time $t$, let $B(t)$ indicate whether that failure would be included in the sample and $\rho_{i}(t)$ be the probability of inclusion if subject $i$ was that failure. Typically, the $\rho_{i}(t)$ will take on a small number of values depending upon subject $i$ 's sampling stratum but, in the development that follows, it is not required. We now redefine $\mathcal{F}_{t-}$ to additionally include the case sampling information in $[0, t)$. Analogous to (5)

$$
\begin{aligned}
& \operatorname{pr}\left\{\tilde{\mathcal{R}}(t)=\mathbf{r}, B(t)=b \mid \Delta N_{i}(t)=1, \mathcal{F}_{t-}\right\}= \\
& \quad \operatorname{pr}\left\{\widetilde{\mathcal{R}}(t)=\mathbf{r} \mid \Delta N_{i}(t)=1, B(t)=b, \mathcal{F}_{t-}\right\} \times \operatorname{pr}\left\{B(t)=b \mid \Delta N_{i}(t)=1, \mathcal{F}_{t-}\right\}
\end{aligned}
$$

The first probability is as in (5) if $b=1$ and $I(\mathbf{r}=\{i\})$ if $b=0$. The second probability is $\rho_{i}(t)$ or $1-\rho_{i}(t)$ for $b=1,0$, respectively. We define $N_{(i, \mathbf{r}, b)}(t)$, for $\mathbf{r} \in \mathcal{P}^{(m)} \cup\{\{i\}\}, i \in \mathbf{r}$, and $b=0,1$, as the number of times in $[0, t]$ a failure of the $i$ th individual is not included, respectively included, in the sample and the sampled risk set equals $\mathbf{r}$. Then the counting processes $N_{(i, \mathbf{r}, b)}$ have intensity processes

$$
\begin{aligned}
\lambda_{(i, \mathbf{r}, b)}(t)= & Y_{i}(t) \lambda_{0}(t) \exp \left\{\beta_{0}^{\prime} Z_{i}(t)\right\} C(t)^{-1} w_{i}(t) \rho_{i}(t) I\left\{\mathbf{r} \in \mathcal{P}_{i}(t), b=1\right\} \\
& +Y_{i}(t) \lambda_{0}(t) \exp \left\{\beta_{0}^{\prime} Z_{i}(t)\right\}\left\{1-\rho_{i}(t)\right\} I(\mathbf{r}=\{i\}, b=0)
\end{aligned}
$$

The partial likelihood may be derived as in (7) - (9) with

$$
\pi_{t}(i \mid \mathbf{r}, 1)=\frac{\exp \left\{\beta_{0}^{\prime} Z_{i}(t)\right\} w_{i}(t) \rho_{i}(t)}{\sum_{j \in \mathbf{r}} \exp \left\{\beta_{0}^{\prime} Z_{j}(t)\right\} w_{j}(t) \rho_{j}(t)}
$$

if $\mathbf{r} \in \mathcal{P}_{i}(t)$ and $\pi_{t}(i \mid \mathbf{r}, 0)=1$ if $\mathbf{r}=\{i\}$.

Thus, letting $\widetilde{F}$ denote the set of failure times for the sampled failures, the partial likelihood for the data may be written in standard notation as

$$
\mathcal{L}(\beta)=\prod_{t_{j} \in \widetilde{F}}\left[\frac{\exp \left\{\beta^{\prime} Z_{i_{j}}\left(t_{j}\right)\right\} w_{i_{j}}\left(t_{j}\right) \rho_{i_{j}}\left(t_{j}\right)}{\sum_{k \in \widetilde{\mathcal{R}}\left(t_{j}\right)} \exp \left\{\beta^{\prime} Z_{k}\left(t_{j}\right)\right\} w_{k}\left(t_{j}\right) \rho_{k}\left(t_{j}\right)}\right] .
$$

The basic likelihood properties are derived in a manner analogous to Appendix 1. 


\section{Discussion}

Counter-matching easily generalizes to accomodate multiple population strata with a different baseline hazard for each stratum. In this case, counter-matching would be performed within population stratum. Also, the exponential form of the relative risk may be replaced by $r\left\{\beta_{0}^{\prime} Z(t)\right\}$ for a general relative risk function $r(\cdot)$ with $r(0)=1$. Further, the expressions apply without change to multiple event data.

Counter-matching itself is more flexible than may be interpreted by our presentation. We have presented the method with sampling strata based on a subject's absolute exposure measure. Another valid method is to define sampling strata based on quantiles of the exposure values of at risk subjects. Counter-matching might also be based on determinants of cost of collecting data. For example, in an occupational cohort of a particular factory, many cohort members may have ceased employment at the factory during the follow up period. The sampling strata could be based on employment status and designed to over-sample those who are still employed since it is easy to contact them. This cannot be done based on present employment status but must be based on employment status at the time of disease being considered so the benefit of this strategy will depend upon how recently the cases of disease generally occurred.

It may well be the situation that the sampling stratum indicator $A$ summarizes a continuous or multilevel covariate $Z_{1}$, which is available on the full cohort, into $L$ sampling strata categories. As mentioned in Section 1, models fitted using the stratified sample which are based just on functions of $A$ retain the full cohort information about $A$. However, since they are more precise, one would typically use the actual values of $Z_{i 1}\left(t_{j}\right)$ when analyzing the stratified sampled data, and the information will be less than that of the full cohort because of the grouping used to define the sampling strata. The question then arises of how to form the sampling strata groupings so as to retain as much information as possible about $Z_{1}$ given that the other covariates are not known. Let $\left(c_{l-1}\left(t_{j}\right), c_{l}\left(t_{j}\right)\right]$ be nonoverlapping intervals with $c_{0}\left(t_{j}\right)=-\infty, c_{L}\left(t_{j}\right)=\infty$ and the sampling strata be defined by $\mathcal{R}_{l}\left(t_{j}\right)=\left\{i \in \mathcal{R}\left(t_{j}\right): c_{l-1}\left(t_{j}\right)<Z_{i 1}\left(t_{j}\right) \leq c_{l}\left(t_{j}\right)\right\}$. We conjecture that a good strategy is to set $L=m$ and choose the $c_{l}\left(t_{j}\right)$ so that the conditional probability of disease, based only on $Z_{1}$, is $1 / m$ in each interval. This depends, of course, on how $Z_{1}$ is modelled and, assuming for example a trend model, may be approximated by choosing the $c_{l}\left(t_{j}\right)$ such that

$$
\frac{\sum_{i \in \mathcal{R}\left(t_{j}\right)} \exp \left\{\hat{\beta} Z_{i 1}\left(t_{j}\right)\right\} I\left\{c_{l-1}\left(t_{j}\right)<Z_{i 1}\left(t_{j}\right) \leq c_{l}\left(t_{j}\right)\right\}}{\sum_{i \in \mathcal{R}\left(t_{j}\right)} \exp \left\{\hat{\beta} Z_{i 1}\left(t_{j}\right)\right\}} \approx 1 / m .
$$

This strategy did appear to be best in a preliminary empirical investigation of the $m=2$ situation but further work is needed to determine if it is generally near optimal.

We have assumed that sampling strata are known for all cohort subjects at the time when the sampling is performed. In many situations, the sampling strata may be available for a portion of the cohort but missing for the rest. Suppose, for ease of exposition, that the sampling strata are based on a dichotomous exposure covariate with the probability of exposure small and that most of the missing values could be filled in for the sampled data. If the number of subjects missing exposure data is small, they could be included with the unexposed forming an "unexposed or missing information" sampling stratum. If there is a large number of such subjects, they could form another sampling stratum and a design with three strata could be considered. In either situation, exposure status would be ascertained for those sampled 
individuals with missing exposure and this would be used in the analysis of the sampled data. The weight associated with such subjects would not depend on their final exposure status but would reflect the sampling stratum from which they were picked.

Counter-matching may also be used when it is not possible to identify all subjects at risk but only to ensure that controls can be randomly sampled from the sampling strata that make up the risk set at a given failure time. This would be the situation in a population based study. Note that the $n_{l}\left(t_{j}\right)$ may be replaced by $\hat{p}_{l}\left(t_{j}\right)=n_{l}\left(t_{j}\right) / n\left(t_{j}\right)$ or by $\hat{p}_{l}\left(t_{j}\right) / \hat{p}_{1}\left(t_{j}\right)$ in the weights without changing the partial likelihood (2). This suggests that if the proportion or ratio of proportions of the population in each sampling stratum is known as a function of time, these methods may be applied.

Finally, if the $\hat{p}_{l}\left(t_{j}\right)$ are not known, they could be estimated from a sample of the population. This could be done in a number of ways. One is to do a survey of the population and estimate the $\hat{p}_{l}\left(t_{j}\right)$ from this sample. Another is to use a two-stage sampling procedure, analogous to that of Breslow and Cain (1988). In this method a "first stage" sample, a random sample of potential "controls" without regard to sampling strata is picked for each case. The sampling stratum of each subject in the first stage sample is determined and the "second stage" counter-matched sample is then picked. The $\hat{p}_{l}\left(t_{j}\right)$ would be estimated from the first stage sample. Further work is needed to assess the validity of these approaches and to develope variance adjustment methods to account for the additional variation resulting from the estimation of the weights.

\section{Acknowledgements}

This research was initiated when the both authors were on sabbatical leave at the MRC Biostatistics Unit, Cambridge, England, the academic year 1991/92. The MRC Biostatistics Unit is acknowledged for its hospitality and for providing us with the best working facilities during this year. The authors wish to thank David Clayton of the Unit for pointing out the importance of this problem. We gratefully acknowledge the support of this work by the Norwegian Research Council for Science and the Humanities and the United States National Cancer Institute.

\section{Appendix 1. Basic likelihood properties of the partial likelihood}

To show that the partial likelihood has basic likelihood properties, first note that (2) may may be written as $\mathcal{L}(\beta, \tau)$, where

$$
\mathcal{L}(\beta, t)=\prod_{u \in[0, t]} \prod_{\mathbf{r} \in \mathcal{P}(u)} \prod_{i \in \mathbf{r}}\left[\frac{\exp \left\{\beta^{\prime} Z_{i}(u)\right\} w_{i}(u)}{\sum_{j \in \mathbf{r}} \exp \left\{\beta^{\prime} Z_{j}(u)\right\} w_{j}(u)}\right]^{\Delta N_{(i, \mathbf{r})}(u)} .
$$

We then introduce the notation

$$
S_{\mathbf{r}}^{(\gamma)}(\beta, t)=\sum_{j \in \mathbf{r}} Z_{j}(t)^{\otimes \gamma} \exp \left\{\beta^{\prime} Z_{j}(t)\right\} w_{j}(t)
$$

for $\gamma=0,1,2$, where for a vector $a, a^{\otimes 0}=1, a^{\otimes 1}=a$ and $a^{\otimes 2}=a a^{\prime}$, and let

$$
E_{\mathbf{r}}(\beta, t)=S_{\mathbf{r}}^{(1)}(\beta, t) / S_{\mathbf{r}}^{(0)}(\beta, t)
$$


and

$$
V_{\mathbf{r}}(\beta, t)=\frac{S_{\mathbf{r}}^{(2)}(\beta, t)}{S_{\mathbf{r}}^{(0)}(\beta, t)}-E_{\mathbf{r}}(\beta, t)^{\otimes 2}
$$

Note that the two quantities $\mathbf{E}_{\mathbf{r}}(\beta, t)$ and $\mathbf{V}_{\mathbf{r}}(\beta, t)$ are the expectation and the covariance matrix, respectively, of the covariate vector $Z_{i}(t)$ if an individual is selected with probability $\pi_{t}(i \mid \mathbf{r} ; \beta) ; c f .(9)$.

Then, apart from a constant term,

$$
\log \mathcal{L}(\beta, t)=\int_{0}^{t} \sum_{\mathbf{r} \in \mathcal{P}(u)} \sum_{i \in \mathbf{r}}\left[\beta^{\prime} Z_{i}(u)-\log \left\{S_{\mathbf{r}}^{(0)}(\beta, u)\right\}\right] \mathrm{d} N_{(i, \mathbf{r})}(u)
$$

Differentiation with respect to $\beta$ yields the "score vector process"

$$
U(\beta, t)=\frac{\partial}{\partial \beta} \log \mathcal{L}(\beta, t)=\int_{0}^{t} \sum_{\mathbf{r} \in \mathcal{P}(u)} \sum_{i \in \mathbf{r}}\left\{Z_{i}(u)-E_{\mathbf{r}}(\beta, u)\right\} \mathrm{d} N_{(i, \mathbf{r})}(u),
$$

and the "information matrix process"

$$
\mathcal{I}(\beta, t)=-\frac{\partial^{2}}{\partial \beta^{2}} \log \mathcal{L}(\beta, t)=\int_{0}^{t} \sum_{\mathbf{r} \in \mathcal{P}(u)} V_{\mathbf{r}}(\beta, u) \mathrm{d} N_{\mathbf{r}}(u)
$$

Using (6), (12) and (13) it is seen that the score process, evaluated at the true parameter vector $\beta_{0}$, equals

$$
U\left(\beta_{0}, t\right)=\int_{0}^{t} \sum_{\mathbf{r} \in \mathcal{P}(u)} \sum_{i \in \mathbf{r}}\left\{Z_{i}(u)-E_{\mathbf{r}}\left(\beta_{0}, u\right)\right\} \mathrm{d} M_{(i, \mathbf{r})}(u),
$$

where, by standard counting process theory (e.g. Andersen et al., 1993, Section II.4.1), the

$$
M_{(i, \mathbf{r})}(t)=N_{(i, \mathbf{r})}(t)-\int_{0}^{t} \lambda_{(i, \mathbf{r})}(u) \mathrm{d} u
$$

are orthogonal local square integrable martingales with predictable variation processes

$$
\left\langle M_{(i, \mathbf{r})}\right\rangle(t)=\int_{0}^{t} \lambda_{(i, \mathbf{r})}(u) \mathrm{d} u
$$

Thus (17) is a vector valued stochastic integral, and therefore a local square integrable martingale. In particular, the expected score is zero; provided that the expectation exists.

The predictable variation process of (17) is, by (6), (18), (19), (12), (13) and (14),

$$
\begin{aligned}
\left\langle U\left(\beta_{0}, \cdot\right)\right\rangle(t) & =\int_{0}^{t} \sum_{\mathbf{r} \in \mathcal{P}(u)} \sum_{i \in \mathbf{r}}\left\{Z_{i}(u)-E_{\mathbf{r}}\left(\beta_{0}, u\right)\right\}^{\otimes 2} \lambda_{(i, \mathbf{r})}(u) \mathrm{d} u \\
& =\int_{0}^{t} \sum_{\mathbf{r} \in \mathcal{P}(u)} V_{\mathbf{r}}\left(\beta_{0}, u\right) S_{\mathbf{r}}^{(0)}\left(\beta_{0}, u\right) C(u)^{-1} \lambda_{0}(u) \mathrm{d} u
\end{aligned}
$$

Moreover, (16) evaluated at $\beta_{0}$ may be written as

$$
\mathcal{I}\left(\beta_{0}, t\right)=\int_{0}^{t} \sum_{\mathbf{r} \in \mathcal{P}(u)} V_{\mathbf{r}}\left(\beta_{0}, u\right) \lambda_{\mathbf{r}}(u) \mathrm{d} u+\int_{0}^{t} \sum_{\mathbf{r} \in \mathcal{P}(u)} V_{\mathbf{r}}\left(\beta_{0}, u\right) \mathrm{d} M_{\mathbf{r}}(u),
$$


where, by (7) and (8), the

$$
M_{\mathbf{r}}(t)=N_{\mathbf{r}}(t)-\int_{0}^{t} \lambda_{\mathbf{r}}(u) \mathrm{d} u
$$

are local square integrable martingales. Therefore, by (8), (12) and (20),

$$
\mathcal{I}\left(\beta_{0}, t\right)=\left\langle U\left(\beta_{0}, \cdot\right)\right\rangle(t)+\int_{0}^{t} \sum_{\mathbf{r} \in \mathcal{P}(u)} V_{\mathbf{r}}\left(\beta_{0}, u\right) \mathrm{d} M_{\mathbf{r}}(u) .
$$

Thus the observed information equals the predictable variation of the score plus a local square integrable martingale. In particular, by taking expectations, assuming that they exist, it follows that the expected information matrix equals the covariance matrix of the score.

\section{Appendix 2. The asymptotic information matrix}

The asymptotic information matrix $\Sigma$ may be obtained as the limit in probability of $n^{-1}\left\langle U\left(\beta_{0}, \cdot\right)\right\rangle(\tau)$; cf. (20).

We briefly outline how $\Sigma$ may be derived under an independent and identically distributed model where $\left(Y_{i}(\cdot), Z_{i}(\cdot), A_{i}(\cdot)\right) ; i=1,2, \ldots, n ;$ are independent copies of $(Y(\cdot), Z(\cdot), A(\cdot))$ with $A(t) \in$ $\{1, \ldots, L\}$. Let $p(t)=P(Y(t)=1)$ and $p_{l}(t)=P(A(t)=l \mid Y(t)=1)$.

For the purpose of investigating asymptotic behavior, we may substitute

$$
\frac{w_{j}(t)}{n}=\frac{n(t)}{n} \frac{n_{A_{j}(t)}(t)}{n(t)} \frac{1}{m_{A_{j}(t)}}
$$

by $p(t) p_{A_{j}(t)}(t) / m_{A_{j}(t)}$ in $n^{-1}\left\langle U\left(\beta_{0}, \cdot\right)\right\rangle(\tau)$. Therefore, by (12), (14) and $(20), n^{-1}\left\langle U\left(\beta_{0}, \cdot\right)\right\rangle(\tau)$ is asymptotically equivalent to

$$
\int_{0}^{\tau}\left\{C(u)^{-1} \sum_{\mathbf{r} \in \mathcal{P}(u)} \tilde{V}_{\mathbf{r}}\left(\beta_{0}, u\right) \widetilde{S}_{\mathbf{r}}^{(0)}\left(\beta_{0}, u\right)\right\} p(u) \lambda_{0}(u) \mathrm{d} u
$$

where $\widetilde{S}_{\mathbf{r}}^{(\gamma)}\left(\beta_{0}, u\right)$ and $\widetilde{V}_{\mathbf{r}}\left(\beta_{0}, u\right)$ are defined as in (12) and (14), but with $w_{j}(t)$ replaced by $p_{A_{j}(t)}(t) / m_{A_{j}(t)}$.

The expression within brackets in (21) is an average over all the $C(t)$ possible samples from the risk set, and therefore converges in probability to its expected value. To be able to write this expected value, and hence the limit in probability of (21) in a convenient form, we introduce mutually independent covariate vectors $Z_{Y, l, j}(t) ; j=1, \ldots, m_{l} ; l=1, \ldots, L$; with $Z_{Y, l, j}(t)$ distributed as $Z(t)$ conditional on $Y(t)=1, A(t)=l$. Furthermore, we let

$$
\operatorname{Cov}\left\{\hat{Z}_{Y}(t) \mid Z_{Y, 1,1}(t), \ldots, Z_{Y, L, m_{L}}(t)\right\}
$$

be the covariance matrix of the covariate vector $\hat{Z}_{Y}(t)$ one obtains by selecting one of the $Z_{Y, l, j}(t)$ with probability

$$
q_{l, j}(t)=\frac{\exp \left\{\beta_{0}^{\prime} Z_{Y, l, j}(t)\right\} p_{l}(t) / m_{l}}{\sum_{k=1}^{L} \sum_{i=1}^{m_{k}} \exp \left\{\beta_{0}^{\prime} Z_{Y, k, i}(t)\right\} p_{k}(t) / m_{k}}
$$

cf. (9) and the remark following (14). Then the limit in probability of (21), i.e. the asymptotic information matrix, may be given as 


$$
\begin{aligned}
& \Sigma= \\
& \int_{0}^{\tau} \mathrm{E}\left[\operatorname{Cov}\left\{\hat{Z}_{Y}(t) \mid Z_{Y, 1,1}(t), \ldots, Z_{Y, L, m_{L}}(t)\right\} \sum_{l=1}^{L} \sum_{j=1}^{m_{l}} \frac{p_{l}(t)}{m_{l}} e^{\beta_{0}^{\prime} Z_{Y, l, j}(t)}\right] p(u) \lambda_{0}(u) \mathrm{d} u .
\end{aligned}
$$

Note that the corresponding formula for simple random sampling of the controls in Goldstein and Langholz (1992) is a special case with $L=1, p_{1}(t)=1$ and $m_{1}=m$.

\section{Appendix 3. Computing asymptotic relative efficiencies}

Consider the set-up described in the beginning of Section 3, and consider the extension of the model (10) where an interaction term is included:

$$
\lambda(t)=Y(t) \lambda_{0}(t) \exp \left(Z_{1} \beta_{1}+Z_{2} \beta_{2}+Z_{1} Z_{2} \beta_{3}\right) .
$$

We will derive the $3 \times 3$ asymptotic information matrix $\Sigma$ for this situation.

Because the $\pi_{i j}=\operatorname{pr}\left(Z_{1}=i, Z_{2}=j \mid Y(t)=1\right)$ are fixed over time, $\int_{0}^{t} p(s) \lambda_{0}(s) \mathrm{d} s$, which is the expected number of failure events for a "baseline" subject, factors out of (24) and may be ignored for relative efficiency comparisons. We will use $G$ to refer to the constant part of $\Sigma$.

We introduce $T=\left(T_{00}, T_{01}, T_{10}, T_{11}\right)$, where $T_{i k}$ is the number of covariate vectors $Z_{Y, l, j}$, defined just above (22), with first component equal $i$ and second component equal $k$. Furthermore, let

$$
D_{i k}=T_{i k} \frac{\pi_{i}}{m_{i}} e^{i \beta_{1}+k \beta_{2}+i k \beta_{3}},
$$

and introduce $D=D_{00}+D_{01}+D_{10}+D_{11}$. We now compute the components of the conditional covariance (22), which we will denote $C_{j k}$. The expectations here are conditional on the same variables as in (22), i.e. they are with respect to the conditional distribution (23). First, letting $\hat{Z}_{j}$ be the $j$ th component of $\hat{Z}_{Y}$, it is easy to see that

$$
\begin{aligned}
& E \hat{Z}_{1}=\left(D_{10}+D_{11}\right) / D \\
& E \hat{Z}_{2}=\left(D_{01}+D_{11}\right) / D
\end{aligned}
$$

and

$$
E \widehat{Z}_{1} \widehat{Z}_{2}=D_{11} / D
$$

Therefore

$$
\begin{aligned}
& C_{11}=E \hat{Z}_{1}\left(1-E \hat{Z}_{1}\right)=\left(D_{10}+D_{11}\right)\left(D_{01}+D_{00}\right) / D^{2} \\
& C_{12}=E \hat{Z}_{1} \widehat{Z}_{2}-E \hat{Z}_{1} E \widehat{Z}_{2}=\left(D_{00} D_{11}-D_{10} D_{01}\right) / D^{2} \\
& C_{13}=E \hat{Z}_{1} \hat{Z}_{2}\left(1-E \hat{Z}_{1}\right)=D_{11}\left(D_{00}+D_{01}\right) / D^{2} \\
& C_{22}=E \hat{Z}_{2}\left(1-E \hat{Z}_{2}\right)=\left(D_{01}+D_{11}\right)\left(D_{00}+D_{10}\right) / D^{2} \\
& C_{23}=E \hat{Z}_{1} \hat{Z}_{2}\left(1-E \hat{Z}_{2}\right)=D_{11}\left(D_{00}+D_{10}\right) / D^{2} \\
& C_{33}=E \hat{Z}_{1} \hat{Z}_{2}\left(1-E \hat{Z}_{1} \hat{Z}_{2}\right)=D_{11}\left(D_{00}+D_{01}+D_{10}\right) / D^{2} .
\end{aligned}
$$

Lastly,

$$
\sum_{l=0}^{1} \sum_{j=1}^{m_{l}} e^{\beta_{0}^{\prime} Z_{Y, l, j}} \frac{\pi_{l}}{m_{l}}=D .
$$


Therefore, with $\mathcal{C}(T)$ the $3 \times 3$ matrix with elements $C_{j k}$ and $D(T)=D$,

$$
G=E\{\mathcal{C}(T) \times D(T)\},
$$

where the expectation is taken with respect to the distribution of $T$. For simple random sampling of the controls, the $Z_{Y, l, j}$ are $m$ independent and identically distributed random vectors distributed as $Z$ conditional on $Y(t)=1$. Thus the four categories defined by $Z_{1}$ and $Z_{2}$ arise randomly and $T$ has a multinomial $\left(\pi_{00}, \pi_{01}, \pi_{10}, \pi_{11} ; m\right)$ distribution. Setting $t_{11}=m-t_{00}-t_{01}-t_{10}$, we then have with $t=\left(t_{00}, t_{01}, t_{10}, t_{11}\right)$ that $G$ for simple nested case- control sampling is given by

$$
\sum_{t_{00}=0}^{m} \sum_{t_{01}=0}^{m-t_{00}} \sum_{t_{10}=0}^{m-t_{00}-t_{01}} \mathcal{C}(t) D(t)\left(\begin{array}{c}
m \\
t_{00} t_{01} t_{10} t_{11}
\end{array}\right) \pi_{00}^{t_{00}} \pi_{01}^{t_{01}} \pi_{10}^{t_{10}} \pi_{11}^{t_{11}}
$$

For counter-matched sampling, the $m_{l}$ vectors $Z_{Y, l, j}$ are, for $l=0,1$, distributed as $Z$ conditional on $Y(t)=1$ and $A(t)=l$. Thus, $T_{01}$ has a binomial $\left(\pi_{01} / \pi_{0}, m_{0}\right)$ distribution and $T_{11}$ has a $\operatorname{binomial}\left(\pi_{11} / \pi_{1}, m_{1}\right)$ distribution. Letting $t_{00}=m_{0}-t_{01}$ and $t_{10}=m_{1}-t_{11}$, we thus have that $G$ for counter-matched sampling is

$$
\sum_{t_{01}=0}^{m_{0}} \sum_{t_{11}=0}^{m_{1}} \mathcal{C}(t) D(t)\left(\begin{array}{l}
m_{0} \\
t_{01}
\end{array}\right)\left(\begin{array}{l}
m_{1} \\
t_{11}
\end{array}\right)\left(\frac{\pi_{01}}{\pi_{0}}\right)^{t_{01}}\left(\frac{\pi_{00}}{\pi_{0}}\right)^{t_{00}}\left(\frac{\pi_{11}}{\pi_{1}}\right)^{t_{11}}\left(\frac{\pi_{10}}{\pi_{1}}\right)^{t_{10}}
$$

To compute the asymptotic relative efficiencies in Section 3 we used the following strategy. First we computed the $3 \times 3$ matrices (25) and (26), which are proportional to the asymptotic information matrix $\Sigma$, with the relevant $\beta$-values. In particular, note that in Section $3.1, \beta_{1}=\beta_{3}=0$, and in Section 3.2, $\beta_{3}=0$. The variance of $\widehat{\beta}_{2}$ in Section 3.1 is then proportional to the inverse of the $(2,2)$ th entry of $G$. In Section 3.2, the variance of $\widehat{\beta}_{1}$ controlling for $Z_{2}$ in the model (10) without interaction is proportional to the $(1,1)$ th entry obtained after inverting the upper left $2 \times 2$ part of $G$, while the variance of $\hat{\beta}_{3}$ is proportional to the $(3,3)$ th entry in $G^{-1}$.

\section{References}

Andersen, P. K., Borgan, Ø., Gill, R. D., and Keiding, N. (1993). Statistical Models Based on Counting Processes. Springer Verlag, New York.

Breslow, N. and Cain, K. (1988). Logistic regression for two stage case-control data. Biometrika 75, $11-20$.

Cain, K. and Breslow, N. (1988). Logistic regression analysis and efficient design for two stage studies. Am. J. Epidemiol. 128, 1198-206.

Breslow, N. E., Lubin, J. H., Marek, P., and Langholz, B. (1983). Multiplicative models and cohort analysis. J. Amer. Statist. Assoc., 78, 1-12.

Cox, D. R. (1972). Regression models and life-tables (with discussion). J. Roy. Statist. Soc. B, 34, $187-220$.

Goldstein, L. and Langholz, B. (1992). Asymptotic theory for nested case-control sampling in the Cox regression model. Ann. Statist. 20, 1903-28.

Langholz, B. and Thomas, D. C. (1991). Efficiency of cohort sampling designs: Some surprising results. Biometrics 47, 1563-71.

Oakes, D. (1981). Survival times: Aspects of partial likelihood (with discussion). Internat. Statist. Rev. 49, 235-64.

Thomas, D. C. (1977). Addendum to: Methods of cohort analysis: Appraisal by application to asbestos mining. By F. D. K. Liddell, J. C. McDonald and D. C. Thomas. J. Roy. Statist. Soc. A 140, 469-91. 
Weinberg, C. and Sandler, D. (1991). Randomized recruitment in case-control studies. Am. J. Epidemiol. 134, 421-32.

Weinberg, C. and Wacholder, S. (1990). The design and analysis of case-control studies with biased sampling. Biometrics, $46963-75$.

White, J. (1982). A two stage design for the study of the relationship between a rare exposure and a rare disease. Am. J. of Epidemiol. 115, 119-28. 